\title{
Successful salvage treatment of refractory recurrence of maxillary sinus carcinoma using image-guided high-dose-rate interstitial brachytherapy
}

\author{
Yin Zhang, MM*, Ning Wu, MD*, Zhipeng Zhao, MS, Qiangian Chen, MM, Guanghui Cheng, MD, PhD \\ Department of Radiation Oncology. China-Japan Union Hospital of Jilin University, Changchun, China \\ *These two authors contributed equally to this work
}

\begin{abstract}
This case report illustrates a treatment effect of image-guided high-dose-rate (HDR) interstitial brachytherapy for refractory recurrence of maxillary sinus carcinoma. A 61-year-old male was previously admitted to another hospital and received surgery because of left maxillary sinus squamous cell carcinoma (SCC) 6 years ago. Tumor regrowth was noted 2 years after the initial radical surgery. The patient accepted local excision again for the recurrence, followed by external beam radiotherapy. Despite salvage treatment with surgery and external irradiation, the lesion expanded as $4.8 \times 4.4 \times 4.0 \mathrm{~cm}^{3}$. Because the patient refused palliative resection, we recommended technique of image-guided HDR interstitial brachytherapy. The total doses of $42 \mathrm{~Gy}$ in 12 fractions were delivered to the whole recurrent tumor. Removal of the recurrent tumor was securely achieved by HDR interstitial brachytherapy, guided with ultrasound. The refractory tumor in the patient healed uneventfully after HDR interstitial brachytherapy without recurrence during 8 months of follow-up. This case is remarkable because the patient experienced complete remission by a safe and practicable method with image-guided HDR interstitial brachytherapy.

J Contemp Brachytherapy 2018; 10, 2: 162-168 DOI: https://doi.org/10.5114/jcb.2018.7560l
\end{abstract}

Key words: HDR interstitial brachytherapy, local recurrence, maxillary sinus carcinoma.

\section{Purpose}

Despite significant progress in treatment of carcinoma in paranasal sinuses, loco-regional recurrence remains the most important cause of failure of initial treatment $[1,2,3]$. The basic method of local treatment of relapse in these patients is surgery or repeated radiotherapy [4,5]. In case of deep lesions in paranasal sinuses, due to high risk of complications in course of application, the use of brachytherapy is marginal till now [6]. Therapeutic researches emphasized the importance of individualizing treatment strategies in patient with a recurrence of disease limited to a local site. In this context, we propose a valuable salvage technique using image-guided high-dose-rate (HDR) interstitial brachytherapy to treat refractory recurrence of maxillary sinus carcinoma after conventional treatment.

\section{Case description}

We encountered a 61-year-old male with recurrence of maxillary sinus carcinoma after initial surgery and exter- nal beam radiotherapy, complaining of pain in the soft tissue over involved paranasal sinus. Six years before being admitted to our clinic, he was diagnosed with squamous cell carcinoma (SCC) of left maxillary sinus (T2N0M0, II stage), and received radical surgery in a vicinity clinic. Two years after the initial radical surgery, the patient underwent local excision again for the loco-regional recurrence. He received external beam radiotherapy with a total dose of $50 \mathrm{~Gy}$ in 25 fractions for the bilateral nasal cavity, ethmoid sinus, and left maxillary sinus. Two years later, the recurrent tumor in the ipsilateral maxillary sinus was diagnosed by positron emission tomography-computed tomography (PET-CT) scan. According to the examination before his visit to our clinic, the complete tumor, with size of $4.8 \times 4.4 \times 4.0 \mathrm{~cm}^{3}$, was located in the pyramid-shaped maxillary sinus cavity (Figure 1 ). Despite recommendation for subsequent palliative resection, the patient refused surgery. He was referred to our clinic for treatment and accepted our proposal of brachytherapy.

Before processing of each treatment, an informed consent was obtained from the patient. This study was
Address for correspondence: Guanghui Cheng, MD, PhD, Department of Radiation Oncology, China-Japan Union Hospital of Jilin University, No. 126 Xiantai Street, Changchun, China, phone: +86 13610712080, fax: +86 431 84995511, « e-mail: chengguanghuifl@163.com
Received: 18.01 .2018

Accepted: 03.03.2018

Published: 30.04 .2018 

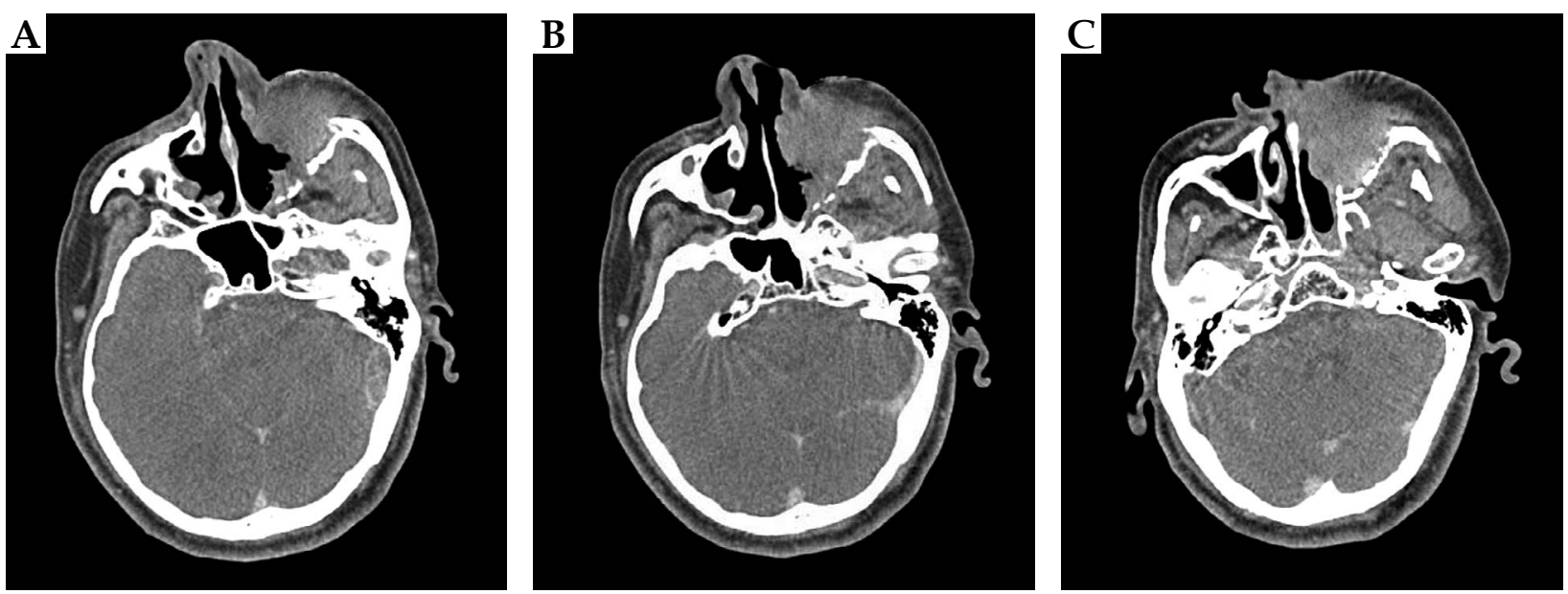

Fig. 1. Recurrent tumor in left maxillary sinus

approved by the China-Japan Union Hospital of Jilin University institutional review board (IRB), and all participants signed an informed consent. HDR interstitial brachytherapy of 42 Gy in 12 fractions for 6 days (3.5 Gy each fraction, twice a day with 6 hours interval, 6 days) was administered to the patient. OncoSmart ProGuide needles (Nucletron, an Elekta company, Elekta AB, Stockholm, Sweden) were used, and the treatment was performed under anesthesia. Electrocardiogram, arterial oxygen pressure, respiration, and blood pressure monitoring were performed during the procedure. Eight applicator catheters $(1.1 \mathrm{~mm}$ in external diameter and $20 \mathrm{~cm}$ in length) were percutaneously inserted into the tumor in a way facilitating irradiation of the whole tumor area under ultrasound guidance, and simultaneously controlling the position of the needle against the adjacent structures. The layout of applicator catheters in the lesion was possibly most parallel, and their distance from one another was about $1 \mathrm{~cm}$ (Figure 2). OncoSmart ProGuide CT-markers were put inside the catheters in order to facilitate their reconstruction. Within $30 \mathrm{~min}$ after implantation of the applicators, fine-pitch $(2 \mathrm{~mm})$ X-ray CT images were then acquired and transferred to the treatment planning computer. The CT-imaging data before interstitial brachytherapy was used to contour gross target volume (GTV) and clinical target volume (CTV). The CTV was expanded from GTV by $10 \mathrm{~mm}$ and restricted by the volume of critical organs (especially eyeball, lens, optic nerve, optic chiasm, and parotid gland). A CT-based treatment plan was created using a graphic optimization tool (treatment planning system Oncentra version 4.3; Nucletron, an Elekta company, Elekta AB, Stockholm, Sweden). The protocol for brachytherapy dose calculations was established based on the dose formalism as defined by an update of the American Association of Physicists in Medicine (AAPM) Task Group No. 43 Report. The normalization and optimization to the target volume was performed.

This report evaluated dosimetry parameters of target volume and organs at risk (OARs) in treatment plan for HDR interstitial brachytherapy using dose volume histogram (DVH) normalization. The dose distribution to target volume and OARs are presented in Figure 3. In the brachytherapy plan, doses were prescribed to nearly $100 \%$ of the target volume. Dosimetry parameters were reported according to the guidelines outlined in ICRU Report 58 [7]. The condition for treatment plan acceptance was encompassing the total CTV, with a dose constituting $100 \%$ and $90 \%$ of the planned dose $\left(D_{100 \%}\right.$ and $D_{90 \%}$, respectively). These doses were converted according to linear quadratic model of biologic effective dose (BED), using the following formula: $B E D=$ nd $(1+d /(\alpha / \beta))$, where $d$ - fractional dose, $n$ - number of fractions, $\alpha / \beta$ - alpha/ beta ratio (in case of SCC of head and neck it is 10). In order to compare them with conventional fractioning of $2 \mathrm{~Gy}$, the equivalent dose for a $2 \mathrm{~Gy}$ fraction schedule was calculated using EQD $\mathrm{ED}_{2}$ model, at $\alpha / \beta=3\left(\mathrm{GyEQD}_{2}, \alpha / \beta=3\right)$ for the OARs, and $\alpha / \beta=10\left(\mathrm{GyEQD}_{2}, \alpha / \beta=10\right)$ for the target. BED and $E Q D_{2}$ were calculated for single fraction and total fractions $\left(\mathrm{BED}-\mathrm{D}_{100 \%}, \mathrm{BED}-\mathrm{D}_{90 \%}, \mathrm{EQD}_{2}-\mathrm{D}_{100 \%}\right.$, and $\mathrm{EQD}_{2}-\mathrm{D}_{90 \%}$, respectively). In order to facilitate the evaluation of correlation between the dose and possible future damages, the volume receiving $100 \%$ of the prescribed dose $\left(\mathrm{V}_{100 \%}\right)$ was reported as well as high dose areas receiving 150\% of the prescribed dose $\left(\mathrm{V}_{150 \%}\right)$ and $200 \%$ of the prescribed dose $\left(\mathrm{V}_{200 \%}\right)$. Dose homogeneity index (DHI), which was used to evaluate the dose uniformity of the target $(\mathrm{DHI}=$ $\left.1-V_{150 \%} / V_{100 \%}\right)$, was calculated for a single fraction [8].

Due to various locations, doses received by OARs were also reported. Depending on the type of the organ, either maximum dose $\left(\mathrm{D}_{\text {max-left eyeball }}, \mathrm{D}_{\text {max-right eyeball }}, \mathrm{D}_{\text {max-left lens }}\right.$ $\mathrm{D}_{\text {max-right lens' }} \mathrm{D}_{\text {max-left optic nerve' }} \mathrm{D}_{\text {max-right optic nerve' }}$ and $\mathrm{D}_{\text {max-optic chiasm' }}$ respectively) or dose per 1 cubic centimeter of the organ located in the maximum dose area $\left(\mathrm{D}_{1 \mathrm{cc}-\text { leftparotidgland }}\right.$ and $\mathrm{D}_{\text {1cc-rightparotidgland }}$, respectively) were reported. The doses were converted to $\mathrm{BED}$ and to $\mathrm{EQD}_{2}$ according to the linear quadratic model $\left(\mathrm{BED}_{- \text {single fraction }}\right.$ $\mathrm{BED}_{\text {-total fractions' }} \mathrm{EQD}_{2 \text {-single fraction' }}$ and $\mathrm{EQD}_{2 \text {-total fractions' }}$ respectively).

Around 2 hours after CT acquisition, the creation of treatment plan for HDR interstitial brachytherapy was completed. After transporting the planning data to an ${ }^{192} \mathrm{Ir}$ remote afterloader system (Microselectron HDR 192Ir; Nucletron, an Elekta company, Elekta AB, Stockholm, Swe- 

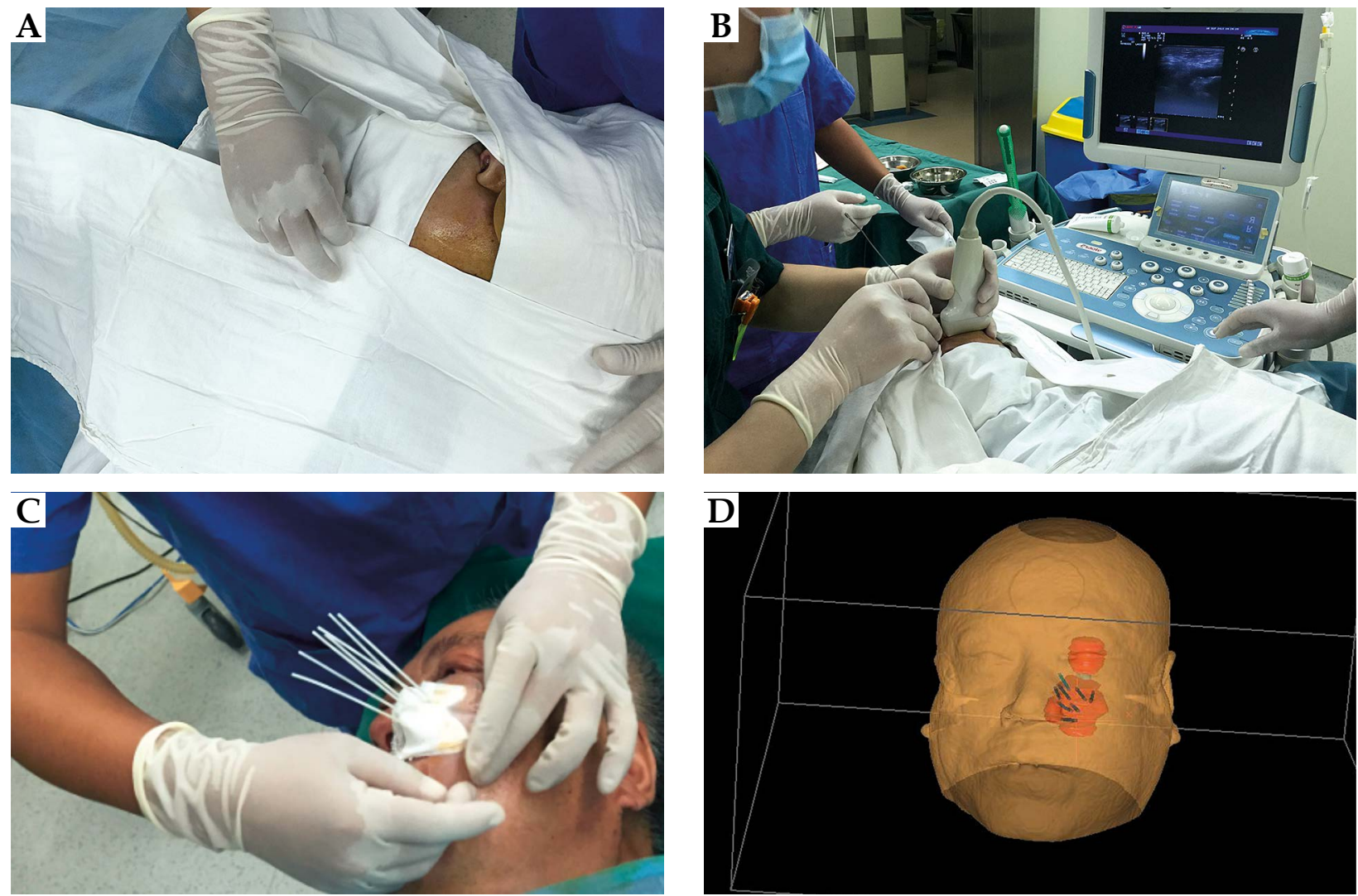

Fig. 2. Eight applicator catheters inserted to the target under ultrasound guidance $(\mathbf{A}, \mathbf{B}, \mathbf{C})$ and reconstructed with three-dimensional mode (D)

den), irradiation procedure was started. The irradiation took approximately $5 \mathrm{~min}$. After completion of irradiation session, applicator catheters were removed from lesions and the patient was discharged after 2 hours of observation. No complications were reported during the treatment and brachytherapy was well tolerated by the patient. The patient is regularly followed up at our affiliated clinics.

\section{Results}

$\mathrm{D}_{100 \%}$ for a single fraction was $2.06 \mathrm{~Gy}$, and $\mathrm{D}_{90 \%}$ for a single fraction was $4.12 \mathrm{~Gy}$. $\mathrm{D}_{100 \%}$ total dose was $24.72 \mathrm{~Gy}$, and $\mathrm{D}_{90 \%}$ total dose was $49.44 \mathrm{~Gy}$. The above-mentioned doses were converted to $\mathrm{BED}$ and $\mathrm{EQD}_{2}$. Biologic effective dose in isodose encompassing the whole irradiated volume $\left(\mathrm{D}_{100 \%}\right)$ of all treatment fractions was $29.81 \mathrm{~Gy}$ and $\mathrm{EQD}_{2}$ was $24.84 \mathrm{~Gy}$. Biologic effective dose in isodose encompassing $90 \%$ of the irradiated volume $\left(D_{90 \%}\right)$ was $69.81 \mathrm{~Gy}$ and $\mathrm{EQD}_{2}$ was $58.17 \mathrm{~Gy}$. DHI was 0.24 and CTV received $100 \%$ dose as well as $150 \%$ and $200 \%$ dose (Table 1). Depending on the location of irradiated area, doses received by OARs were reported (Table 2).

Radiotherapy-related toxicity was measured using radiation therapy oncology group (RTOG) scale [9]. Despite close vicinity of many important structures in the head and neck area, no mechanical injury of these OARs was observed, either in course of the insertion procedure or during the procedure while the applicators were remaining in the site between subsequent treatment sessions.
One month after brachytherapy, it could be observed that the recurrent tumor in left maxillary sinus shrank noticeably with slight radiation-induced skin reaction (RTOG scale grade 1), which was characterized by left lower eyelid edema and skin pigmentation. During the follow-up examination in the $6^{\text {th }}$ month after brachytherapy, the refractory recurrence of maxillary sinus carcinoma disappeared according to the CT results. The pain symptoms decreased, and the related region of skin surface was fully recovered (Figure 4). There are no signs or symptoms of complications and no evidence of recurrence at the site of HDR interstitial brachytherapy throughout over 8 months' observation period.

\section{Discussion}

$70-80 \%$ of tumors of the paranasal sinuses originate in maxillary sinus. For most SCC of the maxillary sinus, the standard treatment is a combination of radical surgery and pre-operative or post-operative irradiation [4,5]. However, loco-regional recurrence remains the most important sign of relapse of disease after treatment of advanced cancer of the maxilla and sinonasal region [1,2]. The recurrence of maxillary sinus carcinoma may be associated with tumor anatomical location, surgical safe zone, and genetic background (e.g., EGFR, etc.) [3,10]. A large number of clinical studies have confirmed that salvage surgery or repeated radiotherapy could be chosen as the local treatment of relapse in these patients $[4,5]$. However, 


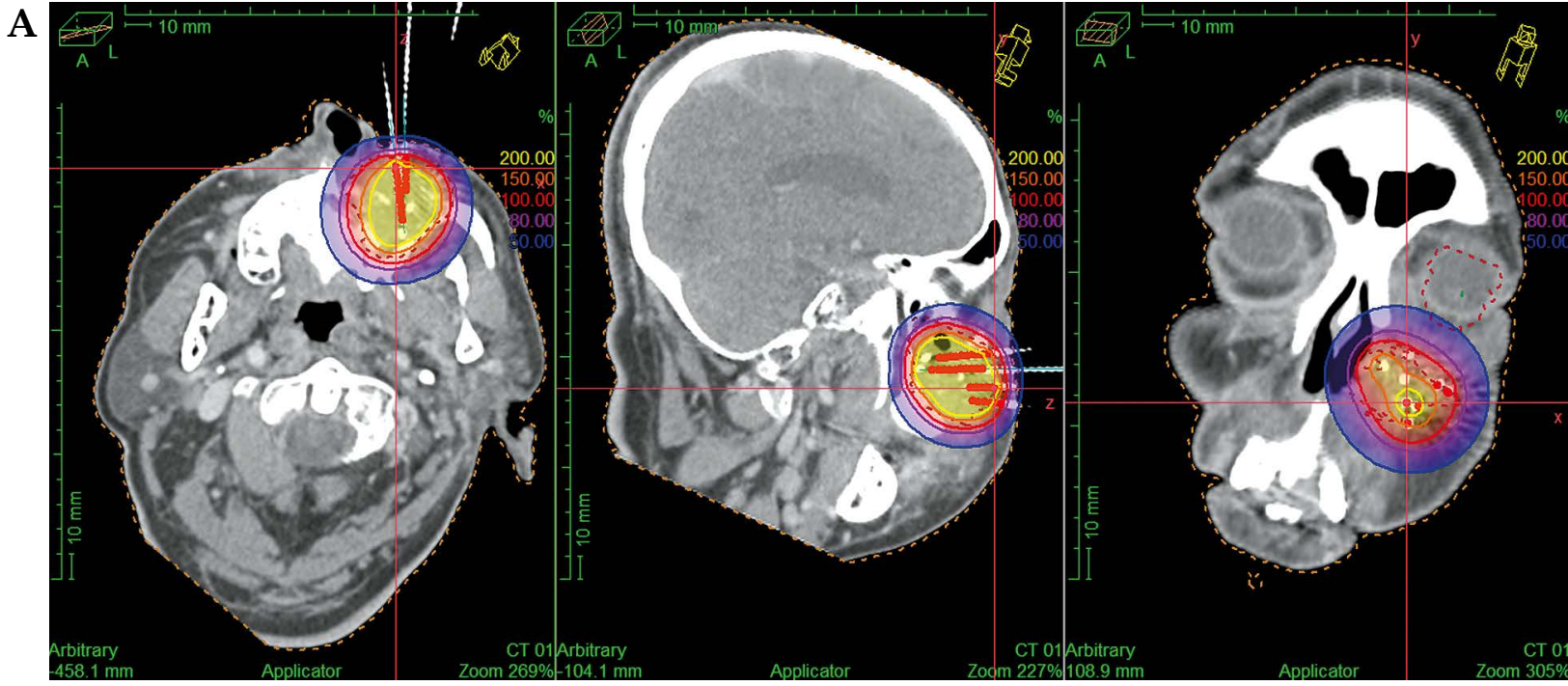

B

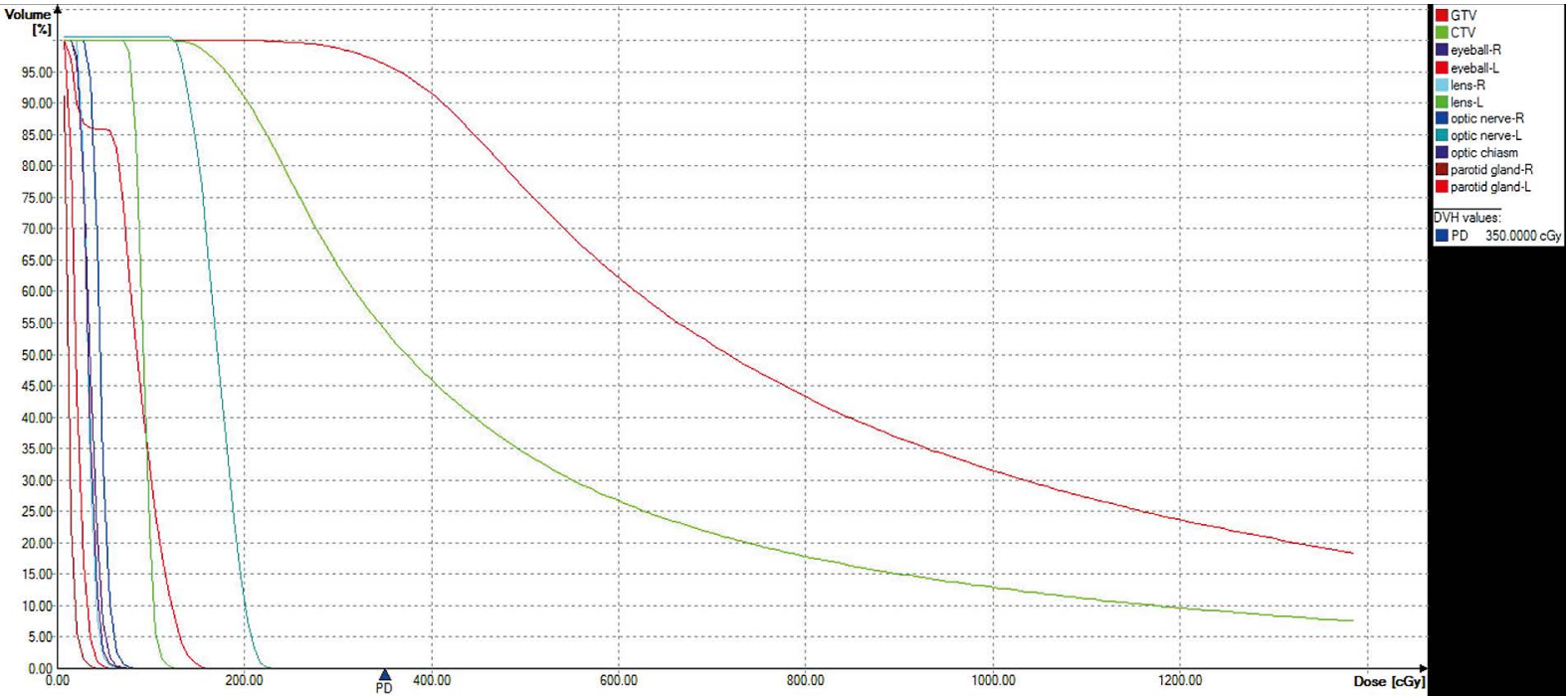

Fig. 3. The dose distribution of (A) horizontal, sagittal, and coronal positions. B) The dose volume histogram (DVH) parameters. It showed high dose distribution of target volume, and low dose distribution of OARs: eyeball, lens, optic nerve, optic chiasm, and parotid gland

Table 1. Doses (Gy) of $D_{100 \%}, D_{90 \%}, B E D-D_{100 \%}, B E D-D_{90 \%}, E Q D_{2}-D_{100 \%}, E Q D_{2}-D_{90 \%}$, and value of $V_{100 \%}, V_{150 \%}$, $\mathrm{V}_{200 \%}$, DHI for target volume

\begin{tabular}{lcccccccccc} 
& $\begin{array}{c}\mathrm{D}_{100 \%} \\
(\mathrm{~Gy})\end{array}$ & $\begin{array}{c}\mathrm{D}_{90 \%} \\
(\mathrm{~Gy})\end{array}$ & $\begin{array}{c}\mathrm{BED}-\mathrm{D}_{100 \%} \\
(\mathrm{~Gy})\end{array}$ & $\begin{array}{c}\mathrm{BED}-\mathrm{D}_{90 \%} \\
(\mathrm{~Gy})\end{array}$ & $\begin{array}{c}\mathrm{EQD}_{2}-\mathrm{D}_{100 \%} \\
(\mathrm{~Gy})\end{array}$ & $\begin{array}{c}\mathrm{EQD}_{2}-\mathrm{D}_{90 \%} \\
(\mathrm{~Gy})\end{array}$ & $\mathrm{V}_{100 \%}$ & $\mathrm{~V}_{150 \%}$ & $\mathrm{~V}_{200 \%}$ & $\mathrm{DHI}$ \\
\hline SFr & 2.06 & 4.12 & 2.48 & 5.82 & 2.07 & 4.85 & 0.96 & 0.73 & 0.52 & 0.24 \\
\hline TFr & 24.72 & 49.44 & 29.81 & 69.81 & 24.84 & 58.17 & - & - & - & -
\end{tabular}

$D_{100 \%}, D_{90 \%}$ - dose covering $100 \%$ and $90 \%$ of target volume; $V_{100 \%}, V_{150 \%}, V_{200 \%}$ - percentage of target volume receiving $100 \%, 150 \%$, and $200 \%$ of prescription dose; $\mathrm{DHI}$ - dose homogeneity index; BED- $D_{100 \%}, B E D-D_{90 \%}$ - biologic effective dose in $D_{100 \%}$ and $D_{90 \%} ; E Q D_{2}-D_{100 \%}$, EQD $-D_{90 \%}-$ dose equivalent $2 G y$ in $D_{100 \%}$ and $D_{90 \%}$; $\mathrm{SFr}$ - single fraction; TFr - total fractions

functional and cosmetic outcomes after surgical treatment for patients with advanced recurrent tumors are far from satisfactory [11]. The complication rate of salvage surgery after intensive chemoradiotherapy for patients with head and neck cancer has been reported to be $11-63 \%$ [12].
Sakashita et al. [11] reported that $18 \%$ of patients with recurrent cancer of the maxillary sinus developed serious operative complications after salvage surgery. An observational study by Iwata et al. determined that 51 patients with local recurrence of nasal or paranasal carcinoma 
Table 2. Doses (Gy) in OARs: eyeball, lens, optic nerve, optic chiasm, and parotid gland

\begin{tabular}{|c|c|c|c|c|c|c|}
\hline & $\mathrm{SFr}$ & $\mathrm{TFr}$ & $\mathrm{BED}_{\text {single fraction }}$ & BED-total fractions & $\mathrm{EQD}_{2^{-} \text {single fraction }}$ & $\mathrm{EQD}_{2^{-} \text {total fractions }}$ \\
\hline$D_{\text {max-left eyeball }}$ & 1.57 & 18.84 & 2.39 & 28.70 & 1.43 & 17.22 \\
\hline $\mathrm{D}_{\text {max-right eyeball }}$ & 0.56 & 6.72 & 0.66 & 7.97 & 0.40 & 4.78 \\
\hline$D_{\text {max-left lens }}$ & 1.01 & 12.12 & 1.35 & 16.20 & 0.81 & 9.72 \\
\hline $\mathrm{D}_{\text {max-right lens }}$ & 0.38 & 4.56 & 0.43 & 5.14 & 0.26 & 3.08 \\
\hline $\mathrm{D}_{\text {max-left optic nerve }}$ & 2.12 & 25.44 & 3.62 & 43.42 & 2.17 & 26.05 \\
\hline $\mathrm{D}_{\text {max-right optic nerve }}$ & 0.60 & 7.20 & 0.72 & 8.64 & 0.43 & 5.18 \\
\hline$D_{\text {max-optic chiasm }}$ & 0.46 & 5.52 & 0.53 & 6.37 & 0.32 & 3.82 \\
\hline $\mathrm{D}_{1 \mathrm{cc}-\text { left parotid gland }}$ & 0.39 & 4.68 & 0.44 & 5.29 & 0.26 & 3.17 \\
\hline $\mathrm{D}_{\text {1cc-right parotid gland }}$ & 0.26 & 3.12 & 0.28 & 3.39 & 0.17 & 2.03 \\
\hline
\end{tabular}

SFr - single fraction; TFr - total fractions; $B E D$-single fraction, $B E D$-total fractions - biologic effective dose of OAR for single fraction and total fractions; EQD2-single fraction, EQD2-total fractions - dose equivalent 2 Gy of OAR for single fraction and total fractions; $D_{\text {max-left eyeball, }} D_{\text {max-right eyeball }}, D_{\text {max-left lens, }}, D_{\text {max-right lens, }}, D_{\text {max-left optic nerve, }} D_{\text {max }}$ right optic nerve, $D_{\text {max-optic chiasm }}$ - maximum dose in left and right eyeball, left and right lens, left and right optic nerve, and optic chiasm; $D_{1 c-l e f t}$ parotid gland, $D_{\text {lcc-right parotid }}$ gland - dose in 1 cc of left and right parotid gland

(maxillary sinus [32/51]) were treated with salvage stereotactic reirradiation. The median overall survival and local control periods after reirradiation were 14.5 and 9.5 months, respectively, and grade 3 or higher adverse events were observed in $23 \%$ of patients [13].

Due to the limited therapeutic options, the management of previously pre-treated refractory recurrent maxillary sinus carcinoma remains a challenging problem. Some exploratory studies have focused on the application of brachytherapy $[14,15,16]$. Huang et al. [17] evaluated their experience with ${ }^{125} \mathrm{I}$ brachytherapy for patients with recurrent or locally advanced maxillary cancers showing positive margins after surgery. They found the method may improve the quality of life of patients with maxillary defects. Nag et al. [18] investigated the feasibility and efficacy of intraoperative HDR brachytherapy as a boost to external beam radiotherapy in primary tumors or as a sole adjuvant treatment in recurrent tumors of the paranasal sinuses. HDR brachytherapy can be safely used to deliver a high radiation dose to locally advanced and recurrent tumors. In another study, Cisek et al. [6] reported the transdermal application of interstitial HDR brachytherapy in one patient treated due to relapsed local tumor in maxillary sinus. The CT examination revealed disease stabilization after treatment completion, and no serious complications of treatment were observed. However, the practical experience of image-guided HDR interstitial brachytherapy for refractory recurrence of maxillary sinus carcinoma in the medical literature is inadequate. In the present case, for the patient with relapsed neoplastic lesion located in a site preventing visually controlled brachytherapy, the application of ultrasound image-guided HDR brachytherapy facilitated the treatment in hardly accessible locations, too close to large nerves or blood vessels. In addition, the DVH showed that target volume was covered with high dose irradiation, but the dosage of OAR was relative low. The patient with refractory recurrence obtained good loco-regional control by the HDR interstitial radiotherapy with image guidance. Similarly, to other studies, no complications occurred during application, and no early toxicity was detected. Futhermore, positive effects were achieved in case of both treatment efficiency and quality of life $[6,17,18]$. Although there is ongoing discussion regarding the best management regarding the optimal treatment procedure for refractory recurrence of maxillary sinus carcinoma, the image-guided HDR interstitial brachytherapy is a safe and practicable salvage treatment.

\section{Conclusions}

The main benefit of HDR interstitial brachytherapy is that a high dose of radiation can be precisely applied to the tumor while simultaneously sparing radiation to healthy tissues. Image-guided HDR interstitial brachytherapy has the potential to be a valuable treatment method for patients with loco-regional refractory relapse of maxillary sinus carcinoma located in sites, preventing standard brachytherapy.

\section{Acknowledgements}

This study was partially supported by grants from the National Natural Science Foundation of China (81201737, 31600679), project of Science and Technology Department of Jilin Province (20090458), project of Health and Family Planning Commission of Jilin Province (2014ZC054), Bethune Special Research of Science and Technology Department of Jilin Province (20160101079JC), Horizontal Project of Jilin University (2015373), Health and Family Planning Commission Foundation of Jilin Province (2014ZC054), Foundation for Natural Science (Bethune Special Project) of Jilin Provincial Science and Technology Department (20160101079JC), Jilin University Enterprise Sponsored Research Foundation (2015YX154), Jilin University Network Experiment Project (VE2015081), Jilin University Undergraduate Education Reform Research Project (2015, 2017XYB080), and Jilin University Norman 

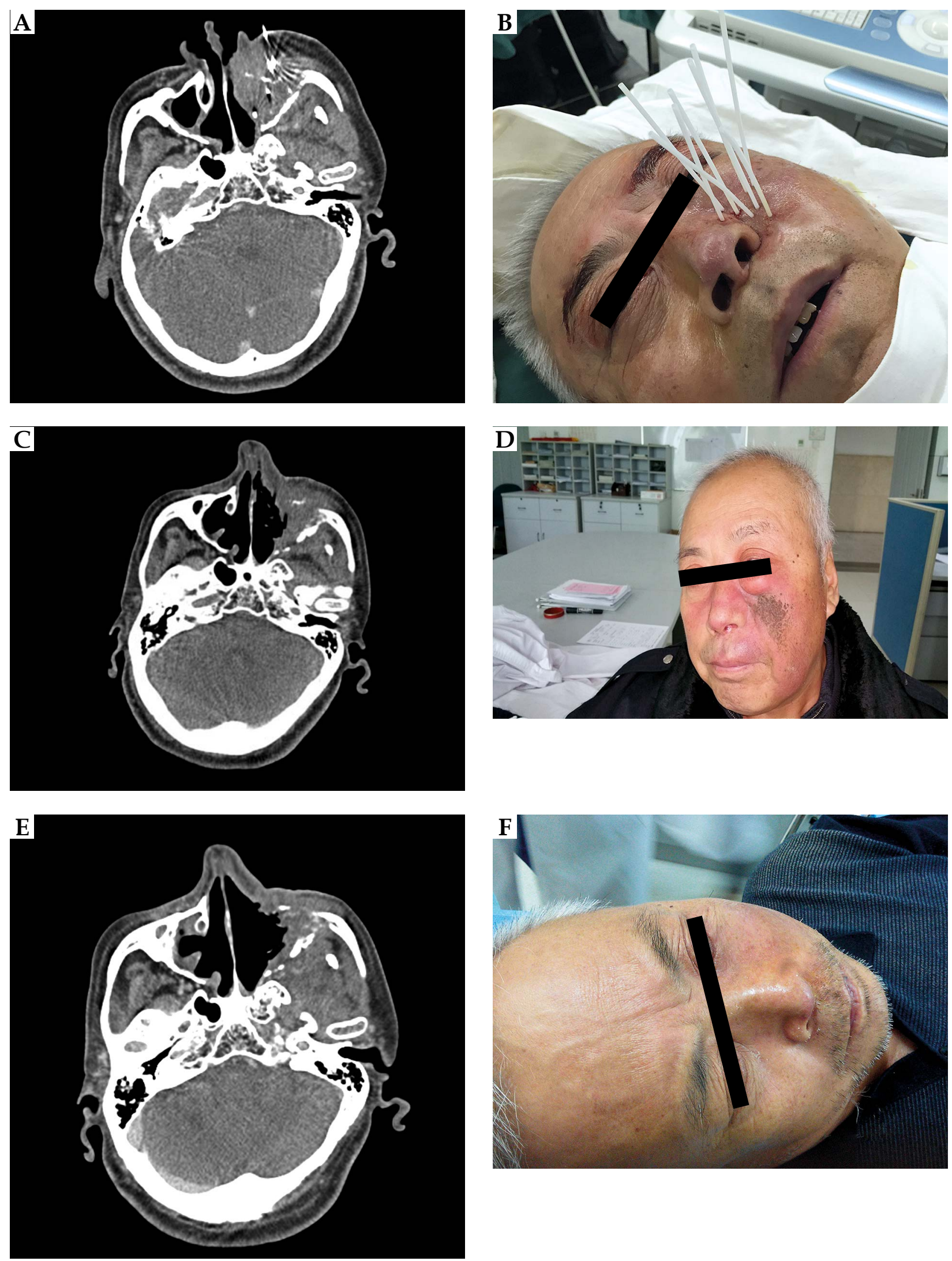

Fig. 4. The recurrent tumor in left maxillary sinus and related region of skin surface (A, B) before, (C, D) 1 month, and (E, F) 6 months after image-guided high-dose-rate interstitial brachytherapy 
Bethune Medical Department Teaching Reform Research Project (B2014B137).

\section{Disclosue}

The authors report no conflict of interest.

\section{References}

1. Snyers A, Janssens GO, Twickler MB et al. Malignant tumors of the nasal cavity and paranasal sinuses: long-term outcome and morbidity with emphasis on hypothalamic-pituitary deficiency. Int J Radiat Oncol Biol Phys 2009; 73: 1343-1351.

2. Thorup C, Sebbesen L, Danø $\mathrm{H}$ et al. Carcinoma of the nasal cavity and paranasal sinuses in Denmark 1995-2004. Acta Oncol 2010; 49: 389-394.

3. McMahon JD, Wong LS, Crowther J et al. Patterns of local recurrence after primary resection of cancers that arise in the sinonasal region and the maxillary alveolus. $\mathrm{Br}$ J Oral Maxillofac Surg 2013; 51: 389-393.

4. Kermer C, Poeschl PW, Wutzl A et al. Surgical treatment of squamous cell carcinoma of the maxilla and nasal sinuses. J Oral Maxillofac Surg 2008; 66: 2449-2453.

5. Bossi P, Farina D, Gatta G et al. Paranasal sinus cancer. Crit Rev Oncol Hematol 2016; 98: 45-61.

6. Cisek P, Kieszko D, Brzozowska A et al. Image-guided highdose-rate brachytherapy of head and neck - a case series study. J Contemp Brachytherapy 2016; 8: 544-553.

7. Das RK. ICRU 58 (Dose and Volume Specification for Reporting Interstitial Therapy) by International Commission on Radiation Units and Measurements. Med Phys 1998; 25: 1225-1225.

8. Wu A, Ulin K, Sternick ES. A dose homogeneity index for evaluating 192 Ir interstitial breast implants. Med Phys 1988; 15: 104-107.

9. Cox JD, Stetz J, Pajak TF. Toxicity criteria of the Radiation Therapy Oncology Group (RTOG) and the European Organization for Research and Treatment of Cancer (EORTC). Int J Radiat Oncol Biol Phys 1995; 31: 1341-1346.

10. Ene P, Popescu RC, Voiculescu $S$ et al. The Role of EGFR and HER2- Activating Mutations in Maxillary Sinus Cancer. Maedica (Buchar) 2012; 7: 70-74.

11. Sakashita T, Homma A, Hatakeyama H et al. Salvage operations for patients with persistent or recurrent cancer of the maxillary sinus after superselective intra-arterial infusion of cisplatin with concurrent radiotherapy. $\mathrm{Br}$ J Oral Maxillofac Surg 2014; 52: 323-328.

12. Tan HK, Giger R, Auperin A et al. Salvage surgery after concomitant chemoradiation in head and neck squamous cell carcinomas - stratification for postsalvage survival. Head Neck 2010; 32: 139-147.

13. Iwata $H$, Tatewaki $K$, Inoue $M$ et al. Salvage stereotactic reirradiation using the CyberKnife for the local recurrence of nasal or paranasal carcinoma. Radiother Oncol 2012; 104: 355-360.

14. Kadah BA, Niewald M, Papaspyrou G et al. Customized individual applicators for endocavitary brachytherapy in patients with cancers of the nasal cavity, sinonasal region and nasopharynx. Eur Arch Otorhinolaryngol 2016; 273: 1543-1547.

15. Ciérvide R, Ramos L, Aristu JJ et al. Use of customized-mold brachytherapy in the management of malignancies arising in the maxillary antrum after maxillectomy: a dosimetric analysis. Brachytherapy 2011; 10: 159-162.

16. Teudt IU, Meyer JE, Ritter M et al. Perioperative image-adapted brachytherapy for the treatment of paranasal sinus and nasal cavity malignancies. Brachytherapy 2014; 13: 178-186.

17. Huang MW, Zhang JG, Tong D et al. Postoperative ${ }^{125} \mathrm{I}$ brachytherapy delivered by digital model obturators for re- current or locally advanced maxillary cancers. Laryngoscope 2012; 122: 2461-2467.

18. Nag S, Tippin D, Grecula J et al. Intraoperative high-doserate brachytherapy for paranasal sinus tumors. Int J Radiat Oncol Biol Phys 2004; 58: 155-160. 